\title{
Motor Imagery did not Improve Strength of Biceps Brachii*
}

\author{
Lanxiang He, Zhijun Tian \\ College of Health Science, Wuhan Sports University, Wuhan, China \\ Email: he-lanxiang@126.com \\ Received 2012
}

\begin{abstract}
Numerous studies have confirmed that motor imagery may result in plastic change in motor system as actual physical activity. However, whether motor imagery can improve muscle strength of the trained persons remains unclear. The aim of this study is to investigate the effect of motor imagery on muscle strength. Totally 12 healthy college students were involved in 4 weeks of mental rehearsal of right upper limb movements (flexion and extension of elbow) during 30 min supervision session three times a week. Electromyogram (EMG) and peak torque of biceps brachii, reaction time of subjects were analyzed. Results showed that no significant change in EMG of biceps brachii was observed during motor imagery. After motor rehearsal for 4 weeks, statistically significant difference in EMG, peak torque and reactivity were not observed $(P>0.05)$ when compared with the baseline data. Therefore, motor imagery could not enhance muscle strength of subjects. Whether mental practice is a valid rehabilitation technique needs to be investigated further.
\end{abstract}

Keywords: Motor Imagery; EMG; Peak Torque; Reaction Time

\section{Introduction}

Motor imagery is a mental process of a movement without any overt movement or without any muscle activation[1-2]. The effects of motor imagery on motor learning and motor recovery have been investigated extensively for many years. Numerous studies have indicated that motor imagery may enhance motor recovery and motor learning, which have many aspects in common. One of possible mechanisms is that motor imagery can result in the activation of motor cortex in brain, which is the same as actual physical activity. However, it is still disputed whether motor imagery can improve motor rehabilitation. Especially, whether motor imagery can enhance muscle strength is still unclear.

In this study, 12 healthy college students were recruited for participating motor imagery of upper limb movement (elbow flexion and extension). The physiological parameters including reaction time, EMG and peak torque of biceps brachii were recorded and analyzed after 4 weeks of motor imagery. This study aims to investigate whether motor imagery can increase muscle strength of participants, which may be helpful for persons with sport injury to improve their muscle strength or delay their muscle atrophy.

\section{Participants and Protocol}

\subsection{Participants}

Totally 12 healthy college students (6 females and 6 males, 21-24 years old) volunteered to participate in this study. During the study, all participants did not perform any physical exercise.

\subsection{Protocol}

At the 1st week of trial, baseline data of EMG, muscle strength,

${ }^{*}$ This research was supported by Wuhan Sports University. and reaction time were collected. Then the participants were instructed to perform motor imagery of right elbow flexion and extension according to the protocol. At the 2nd week of trial, the participants started to perform daily motor imagery for 30 min, three times per week for four consecutive weeks.

During motor imagery process, EMG of biceps brachii was also recorded. At the 6th week of trial, their muscle strength, EMG and reaction time of right biceps were detected again.

\section{Methods and Instruments}

\subsection{Messurement of Muscle Strength}

Participants were seated in a chair. Their right arm was semiflexed and was mounted during all measurements. Strength of biceps brachii was measured concentrically at $60^{\circ}$ per second on an isokinetic dynamometer (Biodex-4, USA). The peak torque (Newton meters, $\mathrm{N}-\mathrm{m}$ ) was recorded and stored on a personal computer for offline analysis.

\subsection{Messurement of Reaction Time}

The reaction time was recorded by reaction time detector (FYS-I, China). The red light signals were given from the front of the subject. The subject pressed the button using his right hand while responding to the red signal. The mean value of three measurements was used for analysis.

\subsection{Messurement of EMG}

Electromyograph (ME6000T16, Finland) was used for collecting EMG data. The surface electrodes attached to the skin over biceps brachii. The skin was cleaned for the adherence of the electrodes and detection of EMG. The raw EMG signals were band-pass filtered $(10-500 \mathrm{~Hz})$ and recorded. The data were collected at $1000 \mathrm{~Hz}$ and analyzed with Mega-Win soft- 
ware.

\subsection{Data Analysis}

The mean power frequency (MPF) and root mean square (RMS) were calculated from the observed EMG. RMS was used as an indicator of the total myoelectric activity. The MPF served to indicate the firing rate of motor units as it is linearly related to the action potential conduction velocity of the muscle fibre[3].

The Student t-test for paired samples ( measures before trial and after trial) was applied using SPSS (version 13.0). The significant difference was considered at the $\mathrm{P}$ value less than $5 \%$.

\section{Results}

EMG signals of biceps brachii was detected motionless situation (Figure 1A), motor imagery (Figure 1B), isometric contraction (Figure 1C), and isotonic contraction (Figure 1D), respectively. EMG activity in motionless situation and motor imagery both were in baseline level. As shown in Table $\mathbf{1}$, surface EMG activity of biceps brachii did not change significantly after motor imagery for 4 weeks $(P>0.05)$. It suggested that, EMG activity of biceps brachii did not exhibit a significant change due to motor imagery of elbow flexion.

Strength of biceps brachii was also measured concentrically at $60^{\circ}$ per second on an isokinetic dynamometer. The peak torque of biceps brachii after motor imagery was bigger than that before motor imagery. The reaction time after motor imagery was shorter than that before motor imagery. As shown in Table 1, no significant difference was achieved $(P>0.05)$.

\section{Discussion}

Learning a motion skill is just to establish a motion conditioned reflex, which includes sensory input, signals integration by brain cortex and signals output to effectors (muscle). The improvement of these factors may be in favor of learning a motion skill.

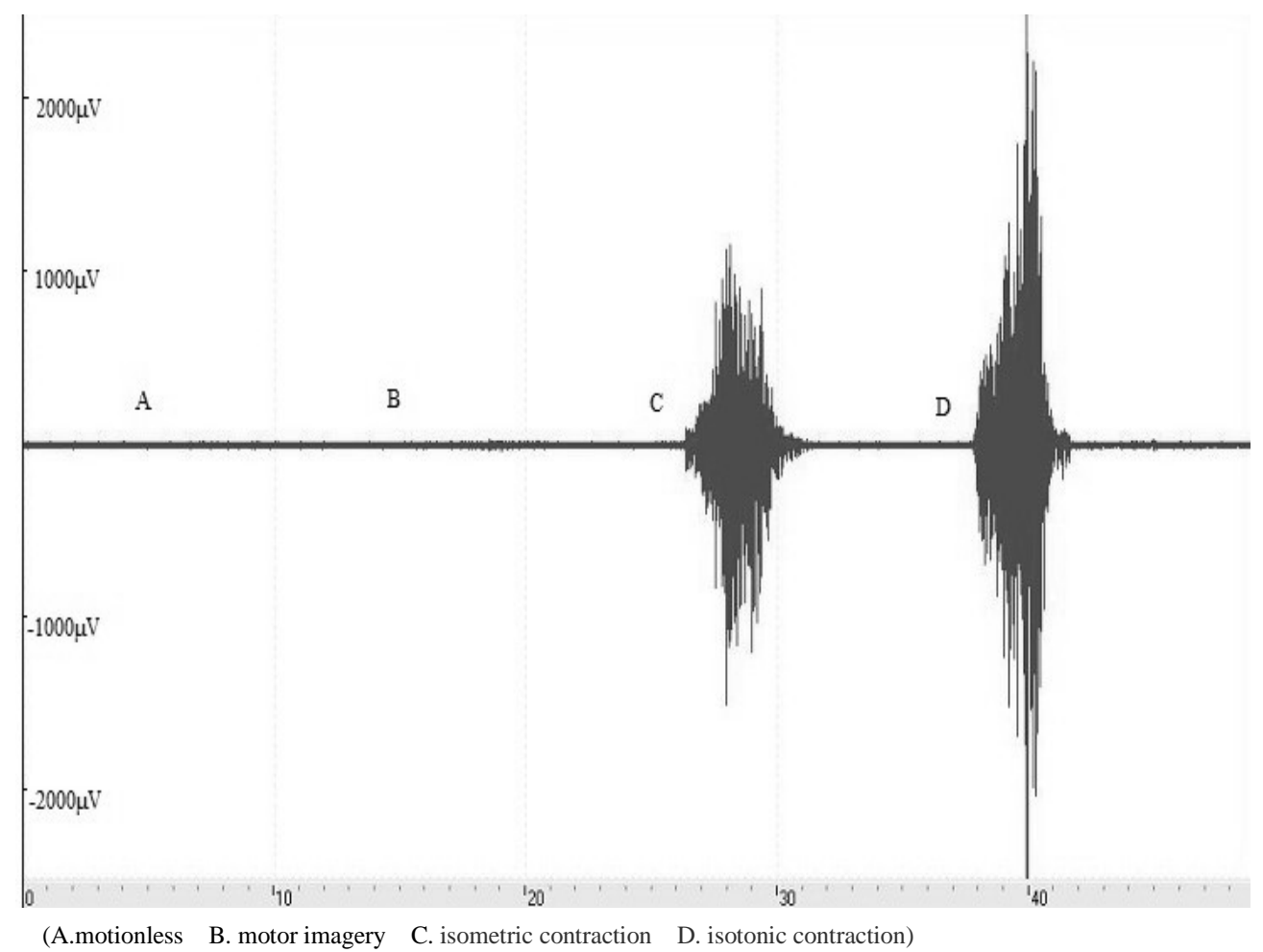

Figure 1. Electromyogram of Bicipital Muscle of Right arm.

Table 1. Reaction Time, Surface EMG and Peak Torque of Biceps Brachii (Mean \pm SD).

\begin{tabular}{|c|c|c|c|c|}
\hline & \multicolumn{2}{|c|}{ Surface EMG } & \multirow{2}{*}{$\begin{array}{l}\text { Peak Torque } \\
\quad \text { (N.m) }\end{array}$} & \multirow{2}{*}{$\begin{array}{l}\text { Reaction Time } \\
\text { (s) }\end{array}$} \\
\hline & $R M S(\mu V)$ & $M P F(\mathrm{~Hz})$ & & \\
\hline Before Trial & $442.1 \pm 187.9$ & $63.2 \pm 6.1$ & $33.57 \pm 12.74$ & $0.3567 \pm 0.0425$ \\
\hline After Trial & $429.7 \pm 169.6$ & $67.0 \pm 9.5$ & $35.82 \pm 12.70$ & $0.3299 \pm 0.0291$ \\
\hline $\mathrm{P}$ & $>0.05$ & $>0.05$ & $>0.05$ & $>0.05$ \\
\hline
\end{tabular}


Brain functional representations and their connections have plasticity [4]. Repeat motion training can stimulate or excite its brain functional representations and strengthen their connections each other, which will be of benefit to sport performance.

Motor imagery is a mental activity. It is a dynamic state during which the representation of a specific motor action is internally activated without any motor output. Increasing evidences have confirmed that both motor imagery and actual physical activity can activate cortical motor areas in the central nervous system. Therefore, the effect of motor imagery on learning motion skill and motor recovery from sport injury has gained tremendous attention.

Previous reports have demonstrated that mental practice can increase its accuracy through ping-pong ball tossing experiment, which suggests that motor imagery may be a useful tool in learning a new motor skill[5]. However, for learning the abduction of the big toe, only subjects who had some experience in the task improved significantly after mental practice as well as after physical practice[6]. For patients, motor recovery is a kind of relearning of motor skill actually. Motor imagery has been also proved to be helpful for motor recovery of patients with stroke[7].

Mechanism of motor imagery is less well understood. Currently, two hypotheses are used for the explanation of motor imagery[8-9]. The central mechanism has made an assumption that motor imagery can stimulate the same cortical areas as actual physical activity and strengthen the connections of relative functional representations, which is supported by functional magnetic resonance imaging (fMRI).

The activation in dorsal premotor cortex, superior parietal lobe and intraparietal sulcus is observed and the cortical representations are overlapped partially during the motor imagery of the participants [10]. Imagery of right-hand finger movements can induce a cortical activation pattern including dorsal and ventral portions of the premotor cortex, frontal medial wall areas, and cortical areas lining the intraparietal sulcus in both cerebral hemispheres[11]. Motor imagery not only has similar functions of neural networks as real physical activity [12], but also can modulate corticomotor excitability[13].

However, a novel continuous pointing method was used to measure and precisely characterize self-motion perception during actual movement and imagined movement through space. The results have revealed that the spatial updating processes that occur during actual self-motion were not evidenced during imagined movement [14].

The peripheral mechanism has supposed that motor imagery may result in neural impulse output so that myoelectrical activity may increase and more fibers may contract synchronously, thus improving muscle strength [15-17]

According to Fontani's report, 30 male participants with motor imagery (karate) have achieved same results in muscle strength improvement as actual training group. However, no obvious change in reactivity is not observed in mental imagery group[16]. Similarly, 11 stroke patients subjected to motor imagery training have greatly improved their limb functions [16]. In contrast, 39 stroke patients involved in mental practice for 4 weeks have revealed inconsistent results [18]. In our study, healthy college students involved in mental practice for 4 weeks also did not exhibit a significant change in surface EMG signal and peak torque when compared with the data before trial, which suggested that 4 weeks of motor imagery training is not enough to enhance muscle strength.

Muscle strength is highly correlated with neural system functions such as modulating velocity and frequency of neural impulse. Faster neural impulse from motor cortex to arm muscles can lead to shorter reaction time. In the present study, motor imagery did not result in the decrease of reaction time, thus suggesting that mental practice has no effect on the modulation of neural impulse velocity. This result is consistent with Fontani's report[16].

In a word, extensive investigations have demonstrated that motor imagery can stimulate cortical functional areas, and the rehabilitation of patients with dyskinesia may benefit from motor imagery [19]. However, the improvement of muscle strength through motor imagery is still lack of convincing evidences. The motor imagery (elbow flexion) for 4 weeks can not improve the strength of biceps in right arm. The optimal training protocol should be further explored in the future.

\section{Acknowledgements}

We are very grateful to Dr. Ning Chen for helpful comments and revise.

\section{REFERENCES}

[1] T. Mulder. "Motor imagery and action observation: cognitive tools for rehabilition,” J Neural Transm, vol.114(10), pp.1265-1278, 2007.

[2] M. Lotze, L.G. Cohen, "Volition and imagery in neurorehabilition,” Cogn Behav Neurol, vol.19(3), pp.135-140, 2006.

[3] A. Lucía, J. Hoyos, J.L. Chicharro, "The slow component of VO2 in professional cyclists,” Br J Sports Med, vol.34, pp.367-374, 2000.

[4] M. Fiori, M. Tinazzi, S.M. Aglioti, "Selective impairment of hand mental rotation in patients with focal hand dystonia," Brain, vol.129(1), pp.47-54, 2006.

[5] J.R. Maring, "Effects of mental practice on rate of skill acquisition,” Phys Ther, vol. 70(3), pp.165-72, 1990.

[6] T. Mulder, S. Zijlstra, W.Zijlstra, J._Hochstenbach, "The role of motor imagery in learning a totally novel movement," Exper Brain Res, vol. 154(2), pp.211-217, 2004.

[7] T. Liu, P. Xu, Q. Yu, DZ. Yao, "The Research and Progress in the Mechanism of Motor Imagery and its Application in Motor Rehabilitation,” Prog.Biochem.Biophys. pp.1-8, 2011.

[8] K.P. Liu, C.C. Chan, T.M. Lee, C.W. Hui-Chan, "Mental imagery for promoting relearning for people after stroke: a randomized controlled trial," Arch Physi Med Rehabil, vol. 85, pp.1403-1408, 2004.

[9] R.Grush, "The emulation theory of representation: motor control, imagery and perception,” Behav Brain Sci, vol. 27(3), pp.377-396, 2004.

[10] F. Filimon, J.D. Nelson, D.J. Hagler, M.I. Sereno, "Human cortical representations for reaching: mirror neurons for execution, observation, and imagery,” Neuroimage, vol. 37(4), pp.1315-1328, 2007.

[11] F.Binkofski, K.Amunts, K.M. Stephan, S. Posse, T. Schormann, H.J. Freund, et al. "Broca's region subserves imagery of motion: a combined cytoarchitectonic and fMRI study," Hum Brain Mapp. vol.11(4), pp.273-285, 2000. 
[12] E. Gerardin, A. Sirigu, S. Lehericy, J.B. Poline, B. Gaymard, C. Marsault, et al. "Partially overlapping neural networks for real and imagined hand movements," Cereb Cortex , vol.10, pp.1093-1104, 2000.

[13] C.M. Stinear, W.D. Byblow, M. Steyvers, O. Levin, S.P. Swinnen, "Kinesthetic, but not visual, motor imagery modulates corticomotor excitability,” Exp Brain Res, vol. 168(1-2) , pp.157-164, 2006.

[14] J.L. Campos, J.H. Siegle, B.J. Mohler, H.H. Bülthoff, J.M. Loomis, "Imagined Self-Motion Differs from Perceived Self-Motion: Evidence from a Novel Continuous Pointing Method," PLoS One, Vol.4(11), pp. e7793-2009.

[15] S. Zhou, Y. Zhang, "Influence of Imagination Training on Muscle Strength and RMS Value of EMG," Journal of Beijing Sport University, vol.27(8), pp.1066-1067, 2004.
[16] G. Fontani, S. Migliorini, R. Benocci, A. Facchini, M. Casini, F. Corradeschi, "Effect of mental imagery on the development of skilled motor actions,” Percept Mot Skills, vol.105, pp.803-826, 2007.

[17] S.J. Page, P. Levine, A. Leonard, "Effects of mental practice on affected limb use and function in chronic stroke," Arch Phys Med Rehabil, vol. 86(3), pp.399-402, 2005.

[18] M. Ietswaart, M. Johnston, H. C. Dijkerman, S. Joice, C. L. Scott, R. S. MacWalter, S. J.C. Hamilton, "Mental practice with motor imagery in stroke recovery: randomized controlled trial of efficacy,” Brain, vol. 134(5), pp. 1373-1386, 2011.

[19] A. Zimmermann-Schlatter, C. Schuster, M.A. Puhan, E. Siekierka, J. Steurer, "Efficacy of motor imagery in post-stroke rehabilitation: a systematic review," J Neuroeng Rehabil, vol. 5, pp.8-10, 2008. 